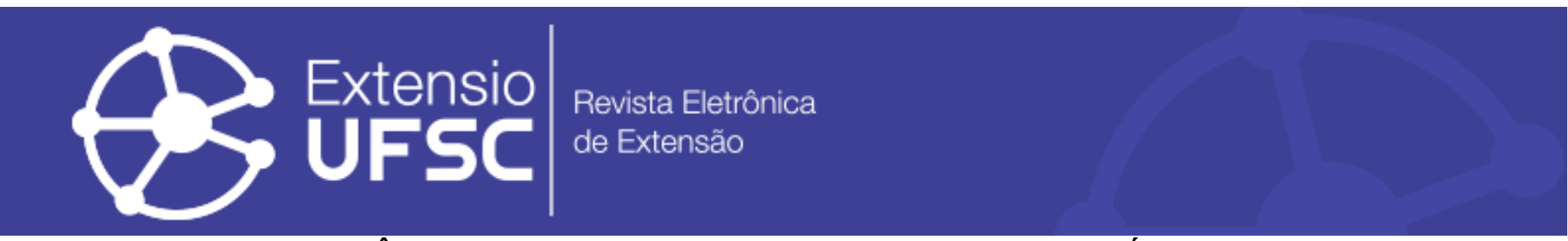

\title{
PREVALÊNCIA E SEVERIDADE DE FLUOROSE DENTÁRIA NA CIDADE DE TETE, MOÇAMBIQUE, NO PERÍODO DE 2013
}

\author{
Ilda Armando Cossa \\ Universidade Federal de Santa Catarina \\ ildalisete@gmail.com \\ Mariane Carvalho Cardoso \\ Universidade Federal de Santa Catarina \\ mariane_cardoso@hotmail.com
}

Resumo

A fluorose dentária é uma doença endêmica, prevalente em cerca de 25 países do mundo. O objetivo deste estudo foi estimar a prevalência e a severidade de fluorose dentária na Cidade de Tete, Moçambique, no período de 2013. Foi feito um estudo epidemiológico, observacional descritivo de corte transversal, com uma amostra representativa de 369 residentes da Cidade de Tete, de 2-34 anos de idade, no período de 2013. Os participantes assinaram o termo de consentimento livre e esclarecido. A avaliação de fluorose dentária foi realizada por dois examinadores treinados e calibrados (inter e intraexaminadores Kappa $>0,7)$ usando Índice de Dean. A prevalência de fluorose dentária foi de 88,1\%, com maior frequência no sexo feminino, em $49,1 \%$. Os grupos de idades mais afetados foram de 5-11 anos, em 20,6\% e de 12-18 anos, em 18,8\%. Houve maior ocorrência de casos leves, nas idades de 5-11 anos em 16,6\% e de casos moderados na faixa etária de 12-18 anos, em 14,5\%. O estudo sugere uma alta prevalência de fluorose dentária, na Cidade de Tete, com predomínio do grau leve, na faixa etária de 5-11 anos, no sexo feminino.

Palavras-chave: Epidemiologia. Fluorose Dentária. Prevalência. Cidade de Tete.

\section{PREVALENCE AND SEVERITY OF DENTAL FLUOROSIS IN THE CITY OF TETE, MOZAMBIQUE, IN 2013}

\begin{abstract}
Dental fluorosis is an endemic disease, prevalent in about 25 countries worldwide. This study aimed to estimate the prevalence and severity of dental fluorosis in the City of Tete, Mozambique, in 2013. An epidemiological, observational descriptive, cross-sectional study was carried out with a representative sample of 369 residents of the City of Tete, aged 2-34 years, in the period of 2013. Participants signed an informed consent form. The assessment of dental fluorosis was performed by two trained and calibrated examiners (inter and intra-examiner Kappa $>0.7$ ) using the Dean Index. The prevalence of dental fluorosis was $88.1 \%$, more frequently in females, at $49.1 \%$. The most affected age groups were $5-11$ years old, in $20.6 \%$, and $12-18$ years old, in 18.8\%. There was a higher occurrence of mild cases, aged 5-11 years in 16.6\% and moderate cases in the age group 12-18 years, in 14.5\%. The study suggests a high prevalence of dental fluorosis in the city of Tete, with a predominance of the mild degree, in the age group of 5-11 years, in females.

Keywords: Epidemiology. Dental Fluorosis. Prevalence. Tete City.
\end{abstract}

\section{PREVALENCIA Y GRAVEDAD DE LA FLUOROSIS DENTAL EN LA CIUDAD DE TETE, MOZAMBIQUE, EN EL PERIODO DE 2013}

\section{Resumen}

La fluorosis dental es una enfermedad endémica que prevalece en unos 25 países en todo el mundo El objetivo de este estudio fue estimar la prevalencia y la gravedad de la fluorosis dental en la Ciudad de Tete, Mozambique, en el período de 2013. Se realizó un estudio epidemiológico, observacional, descriptivo, transversal con una muestra representativa de 369 vecinos del Ayuntamiento de Tete, de 2 a 34 años, en el período de 2013. Los participantes firmaron un consentimiento informado. La evaluación de la fluorosis dental fue realizada por dos examinadores entrenados y calibrados (inter e intraexaminador Kappa > 0,7) utilizando el índice de Dean. La prevalencia de fluorosis dental fue 88,1\%, más frecuentemente en mujeres, 49,1\%. Los grupos de edad más afectados fueron los de 5 a 11 años, en un 20,6\%, y de 12 a 18 años, en un 18,8\%. Hubo una mayor ocurrencia de casos leves, de 5 a 11 años en el 16,6\% y casos moderados en el grupo de edad de 12 a 18 años, en el 14,5\%. El estudio sugiere una alta prevalencia de fluorosis dental en la Ciudad de Tete, con predominio del grado leve, en el grupo de edad de 5-11 años, en el sexo femenino.

Palabras clave: Epidemiología. Fluorosis Dental. Prevalencia. Tete Ciudad. 


\section{INTRODUÇÃO}

A fluorose dentária é definida como um distúrbio de mineralização do esmalte dentário, causado pela intoxicação crônica por flúor, ingerido em grandes concentrações, durante o período de formação e calcificação do esmalte, resultando um esmalte hipoplásico ou hipomineralizado (FEJERSKOV; LARSEN; RICHARDS; BAELUM, 1994; AJIBOYE; DAWSON; FOX, 2018).

É uma doença conhecida em mais de 50 países e endêmica em pelo menos 25 deles, em decorrência dos altos níveis de fluoreto nas fontes naturais de água (FRAZÃO; PERES; CURY, 2011; LIMA; NÓBREGA; CERICATO; ZIEGELMANN et al., 2019). Sendo assim, é um problema de saúde pública, em quase todo o mundo, por causar alterações morfológicas, funcionais e estéticas, que interferem na autoestima e na qualidade de vida dos indivíduos afetados (FEJERSKOV; LARSEN; RICHARDS; BAELUM, 1994; ONORIOBE; ROZIER; CANTRELL; KING, 2014).

Portanto, a Organização Mundial da Saúde (OMS) recomenda que sejam realizados estudos epidemiológicos a cada cinco anos para o controle da fluoração da água e suas reais consequências e benefícios, aplicando-se o índice de Dean para determinar o grau de severidade. O índice classifica a fluorose dentária em questionável, muito leve, leve, moderado ou severo, de acordo com o comprometimento estético do esmalte (WHO, 1997).

As categorias "questionável” e "muito leve" podem não interferir na satisfação com a aparência, de acordo com a cultura de cada região ou nível de perceção de cada indivíduo (AJIBOYE; DAWSON; FOX, 2018). São uma condição esperada e aceite em relação ao risco e benefício na prevenção da cárie dentária através da adição de fluoreto nas águas de abastecimento público, nas concentrações aceitáveis de $0,5-1 \mathrm{mg} \mathrm{F} / \mathrm{L}$ preconizados pela Organização Mundial da Saúde (WHO, 2011; IHEOZOR-EJIOFOR; WORTHINGTON; WALSH; O'MALLEY et al., 2015). Enquanto, as categorias "leve", "moderado" e "severo" são consideradas preocupantes pelo consenso internacional em relação à perceção estética da fluorose dentária (AJIBOYE; DAWSON; FOX, 2018).

A categoria "severa" apresenta, além de comprometimento estético, alterações morfológicas e funcionais, principalmente dos pré-molares e segundos molares (IHEOZOREJIOFOR; WORTHINGTON; WALSH; O'MALLEY et al., 2015). O que interfere na eficiência da mastigação, na autoestima, na socialização do indivíduo e, pode causar dor e exigir tratamento mais complexo (GARCÍA-PÉREZ; IRIGOYEN-CAMACHO; BORGES-YÁÑEZ; ZEPEDAZEPEDA et al., 2017). As formas mais severas são, frequentemente, associadas com a fluoração 
natural das fontes de água, acima do considerado normal (LIMA; NÓBREGA; CERICATO; ZIEGELMANN et al., 2019; MENYA; MAINA; KIBOSIA; KIGEN et al., 2019).

Globalmente, a prevalência e incidência variam de 2,2\% a 90\%. Na África, existem áreas endêmicas na Tunísia, Argélia, Senegal e Marrocos (MENYA; MAINA; KIBOSIA; KIGEN et al., 2019). Na África do Sul, mais de 20\% dos adolescentes apresentam fluorose dentária, maioritariamente em grau muito leve (GROBLERI; LOUW; VAN KOTZE, 2001). Na Nigéria, encontrou-se uma prevalência de 11,3\%, com graus muito leves (OKOYE; EKWUEME; SOTE; AMAECHI, 2019) e há dados de prevalência também na Tanzânia, Gana, Quénia, Etiópia, Uganda, Sudão e Namíbia devido à presença natural de flúor na água potável (FIREMPONG; NSIAH; AWUNYO-VITOR; DONGSOGO, 2013; MENYA; MAINA; KIBOSIA; KIGEN et al., 2019).

Em Moçambique, em estudo realizado com adolescentes de áreas urbanas e suburbanas, na cidade de Maputo, foi encontrada uma prevalência de 8,15\% de fluorose dentária questionável e constatou-se que o único adolescente identificado com fluorose severa era oriundo da Cidade de Tete (MAPENGO, 2010). Contudo, não existem dados documentados nem estudo realizado sobre fluorose dentária nesta Cidade. Além disso, foi observada fluoração natural em fontes de água potável acima de 1,5mg F/L em 8 bairros, dos 9 que compõem a Cidade de Tete. Assim, o objetivo deste estudo foi estimar a prevalência e a severidade da fluorose dentária na Cidade de Tete, para que sejam tomadas medidas preventivas.

\section{MATERIAIS E MÉTODOS}

\section{Considerações éticas}

A pesquisa foi aprovada pelo Comitê de Ética da Faculdade de Ciências de Saúde da Universidade Zambeze, sob o protocolo no 2243/2013, e autorizado pelo Conselho Municipal da Cidade de Tete, pela Direção de Saúde da Cidade e pelos secretários de cada um dos bairros para a realização da mesma. Todos participantes foram informados sobre os procedimentos da pesquisa e garantidos a confidencialidade. E foi obtido o Consentimento Livre e Esclarecido de cada participante e do representante legal dos participantes menores de idade.

\section{Tipo de estudo}


Realizou-se um estudo epidemiológico de tipo observacional descritivo de corte transversal, com a população da Cidade de Tete de 2 a 34 anos de idade, no período de 2013.

\section{População e local do Estudo}

O estudo foi realizado com a população na Cidade de Tete no período de 2013, estimada em 198,097 habitantes, de acordo com o Instituto Nacional da Estatística (INSTITUTO NACIONAL DE ESTATÍSTICA, 2012).

O local de estudo é a capital da província de Tete, localizada na região central e mais ocidental de Moçambique, África Austral. A Cidade de Tete é uma planície que ocupa uma área de $287 \mathrm{~km}^{2}$, atravessados pelo maior rio do país, rio Zambeze. É caraterizado pelo clima semiárido, com temperaturas elevadas, média anual superior a $25^{\circ} \mathrm{C}$ e pluviosidade média anual de $500 \mathrm{~mm}$. A cidade é formada por 9 bairros, alguns no centro da cidade com estradas asfaltadas, sistemas de esgotos e arruamentos ordenados e outros na periferia, com infraestruturas e arruamentos irregulares. Metade dos habitantes tem idade inferior a 18 anos de idade e vive da agricultura em regime familiar, complementada pela criação de gado bovino, caprino e aves. As famílias são constituídas em média por 4 membros. Cerca de $70 \%$ comunica-se com a língua materna e tem pouco conhecimento da língua portuguesa. Os 8 bairros selecionados tinham acesso a sistemas de água comunitários (fontanários), com 59\% de cobertura de abastecimento de água potável, 11\% abastecida por poços/furos e outros com fontes diretamente do rio ou lagos. O flúor na água do consumo era de fonte natural e variava entre 1,3 - >1,5 mg F/L (MINISTÉRIO DA TERRA, AMBIENTE E DESENVOLVIMENTO RURAL, 2015).

\section{Amostra do Estudo}

Para garantir a representatividade da população, foi proposta uma amostragem simples aleatória mediante a fórmula para população finita: $n=(z)^{2} p(1-p) / d^{2}$, onde " $z$ " é o escore da curva normal, cujo valor é 1,96, "p" o parâmetro de referência e "d" é a confiabilidade do estudo em $0,05 \%$.

Dessa forma, obteve-se uma amostra representativa mínima de 369, ao se considerar a ocorrência da doença em $40 \%$ da população. A escolha do parâmetro de 0,40 foi determinada em decorrência de se desconhecer a prevalência da fluorose dentária para a região estudada. E foi acrescentado $10 \%$ para possíveis perdas, totalizando 406 pessoas.

A cidade de Tete é formada por 9 bairros, porém, amostra foi dividida por 8 bairros da cidade, cujas fontes se tinham beneficiado do controle do nível de fluoreto, em janeiro de 2011. Os sujeitos foram selecionados usando o método de amostragem aleatória por conglomerados, em dois estágios. A primeira etapa foi a seleção por ruas e a segunda, a seleção por famílias. Todos os 
participantes de 2 a 34 anos de idade pertencentes às famílias selecionadas foram convidadas a participar do estudo. Para garantir a representatividade de cada bairro e grupos de idades, a amostra foi dividida por igual pelos 8 bairros e depois pelos 4 grupos de idades, resultando 51 pessoas por bairro e 13 pessoas por faixa etária $(2-4,5-11,12-18$ e 19-34 anos). Caso uma família não tivesse todos os grupos de idades, era sorteada outra família, na mesma rua e eram avaliados somente os participantes com as idades em falta. A identificação das famílias foi realizada junto com o secretário de cada bairro.

\section{Critérios de inclusão}

Foram incluídos no estudo os indivíduos com idade compreendida entre os 2-34 anos de idade, nativos que sempre moraram na mesma cidade, residentes nos 8 bairros com registo de níveis de flúor na água do consumo.

\section{Critérios de exclusão}

Foram excluídos do estudo todos aqueles que não eram nativos da cidade, que apresentaram algum tipo de alteração ou incapacidade mental ou física que não permitiu realizar o exame físico bucal, ou que apresentaram ausência ou menos de dois terços da coroa irrupcionada de um dos dentes homólogos mais afetados.

\section{Coleta de dados clínicos}

A avaliação clínica dos participantes foi realizada por dois examinadores treinados e calibrados (inter e intraexaminadores Kappa > 0,7), no ambiente domiciliar, de forma individual, em local reservado e bem iluminado, sentadas em uma cadeira cômoda de frente para o examinador, mediante observação visual direita da cavidade bucal e sob iluminação natural. Os dentes foram secados com compressa estéril, observados com auxílio de espátula de madeira e avaliados quanto à presença e severidade da fluorose. E a informação coletada foi anotada na ficha individual, contendo nome, idade, sexo e o bairro.

Para a avaliação da fluorose dentária foram usados os critérios de índice de Dean (1934), cujo registo é baseado em dois dentes homólogos mais afetado (WHO, 1997).

O índice de Dean modificado recomendado pela OMS permite a comparação entre estudos sobre a fluorose dentária (LIMA; NÓBREGA; CERICATO; ZIEGELMANN et al., 2019).

a) Normal = esmalte translúcido, de superfície lisa, lustrosa, usualmente de cor branco-creme pálida. 
b) Questionável $=$ esmalte com discretas aberrações na translucidez que podem ir desde pequenos traços esbranquiçados até manchas ocasionais. Usado quando a classificação "normal" não se justifica

c) Muito leve = áreas brancas, pequenas e opacas (menos que $25 \%$ da superfície do dente). Inclui opacidades claras com $1 \mathrm{~mm}$ a $2 \mathrm{~mm}$ na ponta das cúspides de molares (picos nevados).

d) Leve $=$ áreas brancas (menos que 50\% da superfície do dente).

e) Moderado $=$ toda a superfície afetada pelas manchas acastanhadas ou amareladas e douradas.

f) Severo $=$ toda a superfície afetada pelas manchas acastanhadas, erosão dentária e hipoplasia do esmalte. E tomaram-se fotografias de casos mais relevantes de fluorose.

\section{Análise dos Dados}

Os dados foram digitados e processados em planilha eletrónica Excel® da Microsoft 365. Posteriormente, foram organizados na forma de tabelas e gráficos, em números absolutos e percentuais. A prevalência da fluorose dentária foi estimada no intervalo de confiança de $95 \%$ e margem de erro de 5 pontos percentuais.

\section{RESULTADOS}

Das 406 pessoas examinadas, 37 que apresentaram o grau questionável da fluorose dentária foram excluídas, para se evitar superestimação ou subestimação da doença. Uma vez que este grau só é aplicável quando não é possível classificar o esmalte como normal, devido às pequenas diferenças em relação a sua translucidez normal (RIPA, 1991; DEMELASH; BEYENE; ABEBE; MELESE, 2019).

A amostra final foi composta por 369 participantes. E a prevalência de fluorose dentária na população estudada foi de $88,1 \%$ (Tabela 1 ). 
Prevalência e severidade de fluorose dentária na cidade de Tete, Moçambique, no período de 2013

Tabela 1 - Distribuição da fluorose dentária na população da Cidade de Tete, por sexo, no período de 2013 (n=369).

\begin{tabular}{ccccccc}
\hline & \multicolumn{2}{c}{ Feminino } & \multicolumn{2}{c}{ Masculino } & \multicolumn{2}{c}{ Total } \\
Fluorose dentária & $\mathrm{n}$ & $\%$ & $\mathrm{n}$ & $\%$ & $\mathrm{n}$ & $\%$ \\
\cline { 2 - 7 } Sim & 181 & 49,1 & 144 & 39,0 & 325 & 88,1 \\
Não & 27 & 7,3 & 17 & 4,6 & 44 & 11,9 \\
Total & 208 & 56,4 & 161 & 43,6 & 369 & 100,0 \\
\hline
\end{tabular}

Fonte: elaborado pelos autores

Houve maior ocorrência no sexo feminino (49,1\%) (Tabela 1). O grupo mais afetado foi de 5-11 anos de idade, em 20.6\%, enquanto o grupo de 2-4 anos de idade apresentou menor número de casos de fluorose dentária, em 4\% (Gráfico 1).

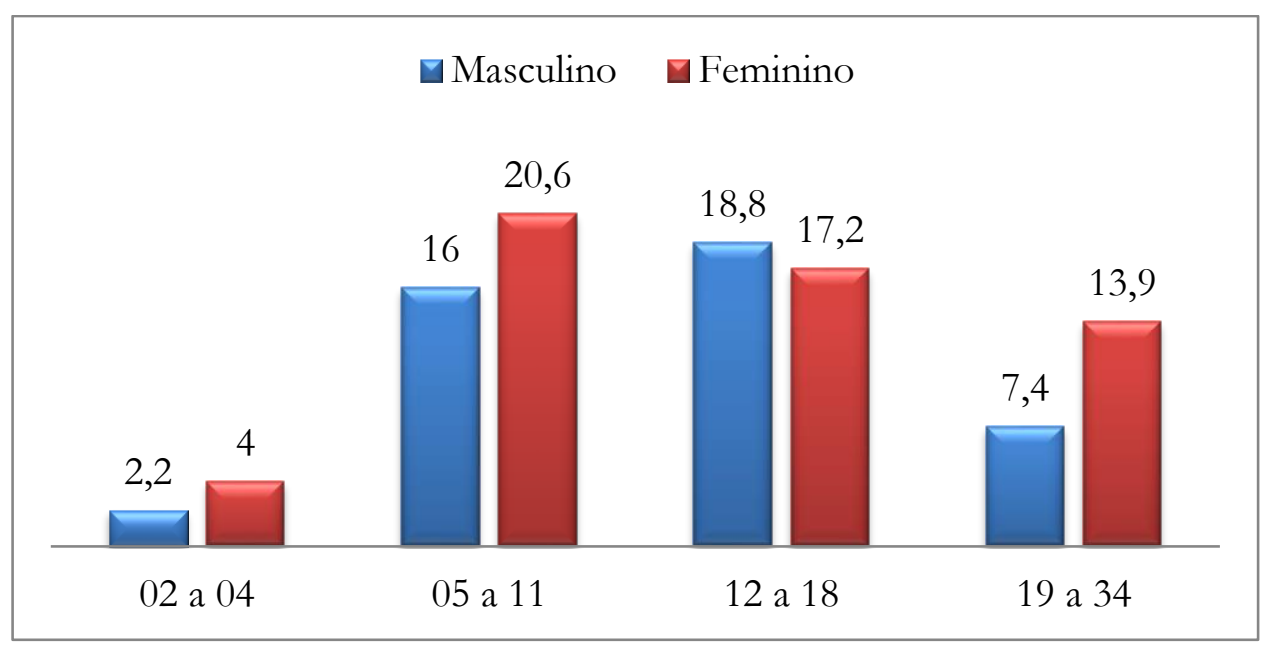

Figura 1 - Distribuição da fluorose dentária na população da Cidade de Tete, por sexo e grupo de idades, no período de 2013 (n=369). Fonte: elaborado pelos autores.

Foi observada maior frequência de casos leves no grupo de 5-11 anos de idade, em 16,6\%, e de casos moderados na faixa etária de 12-18 anos, em 14,5\% (Gráfico 2). 


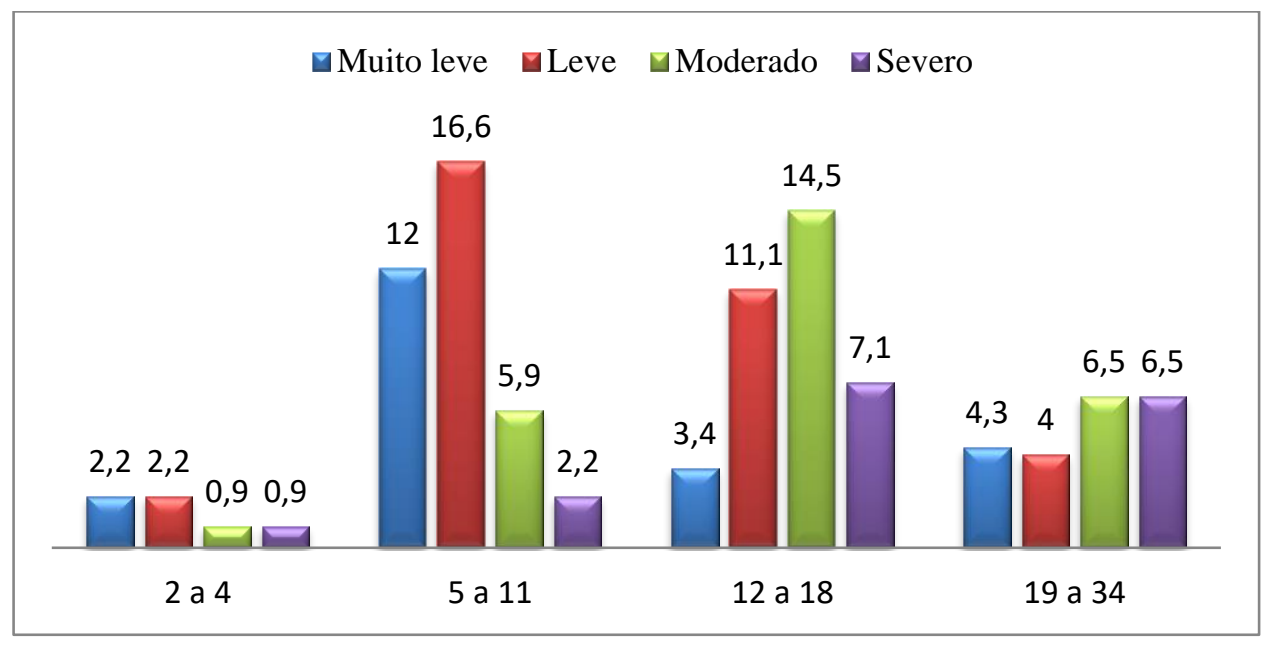

Figura 2 - Distribuição da severidade da fluorose dentária na população da Cidade de Tete, por grupo de idades no período de 2013 ( $\mathrm{n}=369$ ). Fonte: elaborado pelos autores.

O sexo feminino apresentou mais casos leves de fluorose dentária $(18,5 \%)$ do que o sexo masculino (15,4\%) (Gráfico 3).

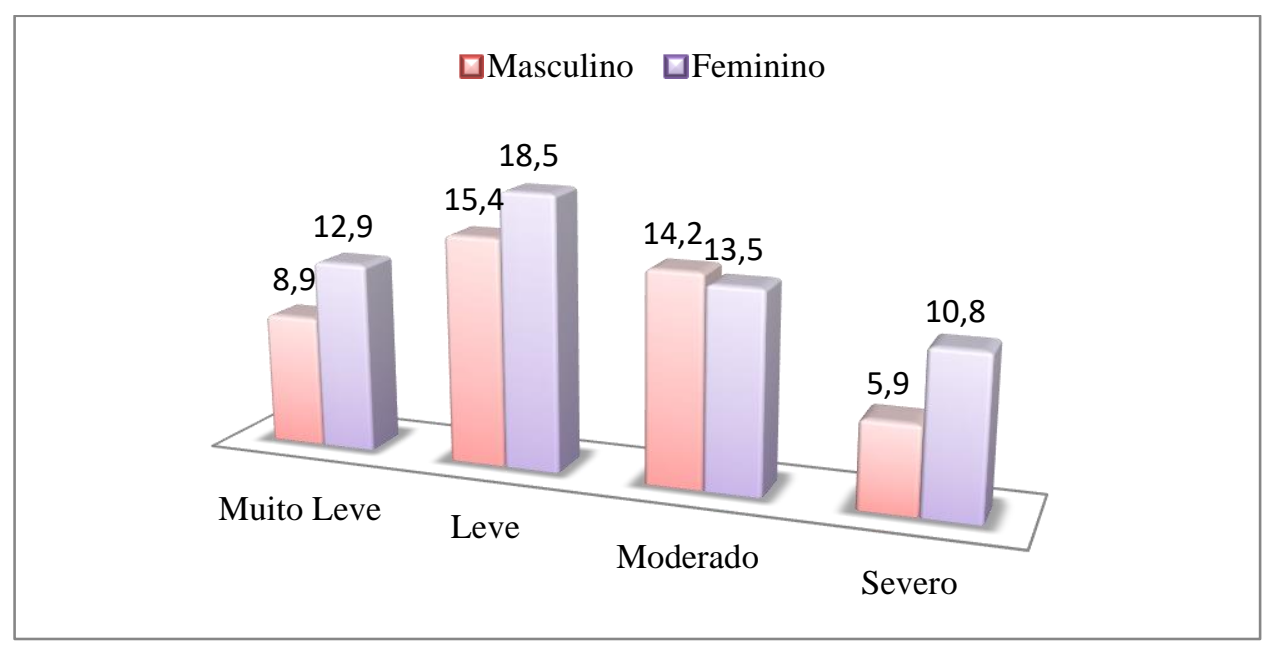

Figura 3 - Distribuição da severidade da fluorose dentária na população da Cidade de Tete, por sexo no período de 2013 (n=369). Fonte: elaborado pelos autores.

\section{DISCUSSÃO}

A prevalência da fluorose dentária na população da Cidade de Tete foi alta. O que sugere, de acordo com as principais causas da doença, a exposição ao flúor em concentrações elevadas na água do consumo (LIMA; NÓBREGA; CERICATO; ZIEGELMANN et al., 2019; ONIPE; EDOKPAYI; ODIYO, 2020).

A OMS preconiza os valores entre $0,5-1 \mathrm{mg} F / \mathrm{L}$ como concentrações normais de fluoreto na água do consumo (WHO, 2011). Já a Associação Dentária Americana aceita os teores de 0,7 - 
1,2 mg F/L, em função das condições climáticas, o nível do mar, média das temperaturas máximas diárias e a frequência de ingestão de água nessa região (FRAZÃO; PERES; CURY, 2011). Conforme estudos realizados, a fluorose dentária tem sido relacionada com a presença de flúor na água de abastecimento público, acima do nível ótimo (FEJERSKOV; LARSEN; RICHARDS; BAELUM, 1994; FIREMPONG; NSIAH; FRAZÃO; PERES; CURY, 2011; AWUNYO-VITOR; DONGSOGO, 2013; MOIMAZ; SALIBA; MARQUES; GARBIN et al., 2015).

Portanto, a alta prevalência de fluorose dentária na população estudada pode ser explicada pela fluoração natural da água do consumo acima do normal $(1,3$ - >1,5 mg F/L), registada nos 8 bairros da Cidade. Resultado esse que corrobora com achados de outros estudos que verificaram maiores prevalências de fluorose dentária de até 100\% em regiões com fluoração natural entre 1,2 - 4,8 mg F/L (DEMELASH; BEYENE; ABEBE; MELESE, 2019; LIMA; NÓBREGA; CERICATO; ZIEGELMANN et al., 2019). Enquanto em regiões com baixos níveis de flúor entre 0,05 a 0,4 mg F/L, foram associados à baixa ocorrência de fluorose dentária (MAPENGO, 2010).

As faixas etárias mais afetadas foram de 5-11 anos e 12-18 anos. que coincidem com as idades de início e término da erupção da dentição permanente. Alguns estudos verificaram que a maior prevalência e severidade eram comuns em populações mais jovens, visto que os seus tecidos estavam em fase do desenvolvimento (GOODARZI; MAHVI; HOSSEINI; NEDJAT et al., 2016; VERMA; SHETTY; GUDDATTU; CHOURASIA et al., 2017). Período esse durante o qual o esmalte pode ser afetado pelo fluoreto, mesmo se nos estágios anteriores não tenha havido exposição. Além disso, as duas faixas etárias incluem as idades índices, 5 e 12 anos, preconizadas pela OMS para estudos epidemiológicos da severidade de fluorose (WHO, 1997).

Houve predomínio do grau leve, categoria que corrobora com os dados epidemiológicos de outros estudos que, de uma forma geral, observaram esta categoria como a condição mais prevalente (MOIMAZ; SALIBA; MARQUES; GARBIN et al., 2015; DEMELASH; BEYENE; ABEBE; MELESE, 2019). Categoria considerada preocupante em relação à perceção estética da fluorose dentária (AJIBOYE; DAWSON; FOX, 2018).

O estudo teve limitações que devem ser reconhecidas. A utilização de luz natural e ausência de profilaxia podem ter dificultado o diagnóstico e, portanto, a classificação do grau de fluorose dentária, atribuído um grau de subjetividade.

Outros estudos epidemiológicos mais detalhados sobre fatores de risco deviam ser realizados nesta população para identificação de fatores de risco associados e implementação de medidas preventivas. 


\section{CONCLUSÃO}

O estudo sugere uma alta prevalência de fluorose dentária, na Cidade de Tete, de grau leve, maioritariamente na faixa etária de 5-11 anos, no sexo feminino.

\section{REFERÊNCIAS}

AJIBOYE, A. S.; DAWSON, D. R., 3RD; FOX, C. H. American Association for Dental Research Policy Statement on Community Water Fluoridation. Journal of Dental Research, v. 97, n. 12, p. 1293-1296, Nov. 2018.

AZAMI-AGHDASH, S.; GHOJAZADEH, M.; POURNAGHI AZAR, F.; NAGHAVIBEHZAD, M. et al. Fluoride concentration of drinking waters and prevalence of fluorosis in iran: a systematic review. J Dent Res Dent Clin Dent Prospects, v.7, n. 1, p. 1-7, fev 2013.

BELTRÁN-AGUILAR, E. D.; BARKER, L.; DYE, B. A. Prevalence and severity of dental fluorosis in the United States, 1999-2004. NCHS Data Brief, n. 53, p. 1-8, nov 2010.

DEMELASH, H.; BEYENE, A.; ABEBE, Z.; MELESE, A. Fluoride concentration in ground water and prevalence of dental fluorosis in Ethiopian Rift Valley: systematic review and metaanalysis. BMC Public Health, v. 19, n. 1, p. 1298, oct 162019.

FEJERSKOV, O.; LARSEN, M. J.; RICHARDS, A.; BAELUM, V. Dental tissue effects of fluoride. Adv Dent Res, v. 8, n. 1, p. 15-31, jun 1994.

FIREMPONG, C.; NSIAH, K.; AWUNYO-VITOR, D.; DONGSOGO, J. Soluble fluoride levels in drinking water-a major risk factor of dental fluorosis among children in Bongo community of Ghana. Ghana Med J, v. 47, n. 1, p. 16-23, mar 2013.

FRAZÃO, P.; PERES, M. A.; CURY, J. A. Drinking water quality and fluoride concentration. Rev Saude Publica, v. 45, n. 5, p. 964-973, oct 2011.

GARCÍA-PÉREZ, Á.; IRIGOYEN-CAMACHO, M. E.; BORGES-YÁÑEZ, S. A.; ZEPEDAZEPEDA, M. A. et al. Impact of caries and dental fluorosis on oral health-related quality of life: a cross-sectional study in schoolchildren receiving water naturally fluoridated at above-optimal levels. Clin Oral Investig, v. 21, n. 9, p. 2771-2780, dec 2017.

GOODARZI, F.; MAHVI, A. H.; HOSSEINI, M.; NEDJAT, S. et al. The prevalence of dental fluorosis and exposure to fluoride in drinking water: A systematic review. J Dent Res Dent Clin Dent Prospects, v. 10, n. 3, p. 127-135, aug 2016.

GROBLERI, S. R.; LOUW, A. J.; VAN KOTZE, T. J. Dental fluorosis and caries experience in relation to three different drinking water fluoride levels in South Africa. Int J Paediatr Dent, v. 11, n. 5, p. 372-379, sep 2001.

IHEOZOR-EJIOFOR, Z.; WORTHINGTON, H. V.; WALSH, T.; O'MALLEY, L. et al. Water fluoridation for the prevention of dental caries. Cochrane Database Syst Rev, v. 18, n. 6, p. Cd010856, Jun 2015. 
INSTITUTO NACIONAL DE ESTATÍSTICA. Anuário Estatístico - Instituto Nacional de Estatística 2011. Disponível em: http://www.ine.gov.mz/estatisticas/publicacoes/anuario/nacionais/anuario-estatistco-statisticalyearbook-2011/view. Acesso 13 jul 2021.

LIMA, I. F. P.; NÓBREGA, D. F.; CERICATO, G. O.; ZIEGELMANN, P. K. et al. Prevalence of dental fluorosis in regions supplied with non-fluoridated water in the Brazilian territory: a systematic review and meta-analysis. Cien Saude Colet, v. 24, n. 8, p. 2909-2922, aug 2019.

MAPENGO, M. A. A. Condições de saúde bucal em adolescentes de Maputo, Moçambique. Dissertação de Mestrado - Faculdade de Odontologia de Bauru. Universidade de São Paulo, Bauru, 2010.

MENYA, D.; MAINA, S. K.; KIBOSIA, C.; KIGEN, N. et al. Dental fluorosis and oral health in the African Esophageal Cancer Corridor: Findings from the Kenya ESCCAPE case-control study and a pan-African perspective. Int J Cancer, v. 145, n. 1, p. 99-109, jul 12019.

MINISTÉRIO DA TERRA, AMBIENTE E DESENVOLVIMENTO RURAL. Perfil ambiental distrital de Cidade de Tete. Disponível em: https://biofund.org.mz/wpcontent/uploads/2019/01/1547451805Copy\%20of\%2015065_2014_F3_PA14_001_0_CTete.pd f. Acesso em: 13 jul 2021.

MOIMAZ, S. A.; SALIBA, O.; MARQUES, L. B.; GARBIN, C. A. et al. Dental fluorosis and its influence on children's life. Braz Oral Res, v. 29, n. 1, p. 1-7, jan 2015.

OKOYE, L. O.; EKWUEME, O. C.; SOTE, E. O.; AMAECHI, B. T. Prevalence of dental fluorosis among 12-15-year-old students in Enugu Metropolis, Nigeria. Indian J Dent Res, v. 30, n. 3, p. 462-467, may./jun 2019.

ONORIOBE, U.; ROZIER, R. G.; CANTRELL, J.; KING, R. S. Effects of enamel fluorosis and dental caries on quality of life. J Dent Res, v. 93, n. 10, p. 972-979, oct 2014.

RIPA, L. W. A critique of topical fluoride methods (dentifrices, mouthrinses, operator-, and selfapplied gels) in an era of decreased caries and increased fluorosis prevalence. J Public Health Dent, v. 51, n. 1, p. 23-41, feb 1991.

SHEKAR, C.; CHELUVAIAH, M. B.; NAMILE, D. Prevalence of dental caries and dental fluorosis among 12 and 15 years old school children in relation to fluoride concentration in drinking water in an endemic fluoride belt of Andhra Pradesh. Indian J Public Health, v. 56, n. 2, p. 122128, Apr-Jun 2012.

WORLD HEALTH ORGANIZATION - WHO. Guidelines for Drinking-water Quality fourth edition. $2011 . \quad$ Geneva: Disponível em: http://whqlibdoc.who.int/publications/2011/9789241548151-eng.pdf. Acesso 12 feb 2012.

WORLD HEALTH ORGANIZATION. Oral health surveys: basic methods. 4. ed. geneva: WHO, 1997. p. 66. APÊNDICES I - Consentimento informado

Recebido em: 02/07/2020

Aceito em: 10/08/2021 
Prevalência e severidade de fluorose dentária na cidade de Tete, Moçambique, no período de 2013

\section{ANEXOS}

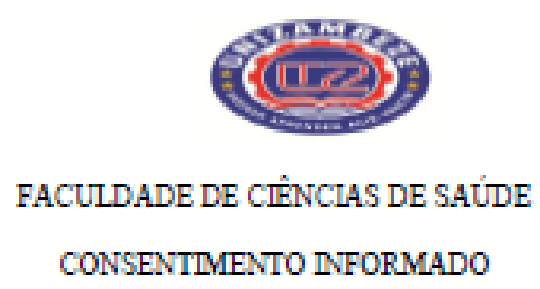

Ex. Sr. Encarregado de Educação / Participante

O seu educando foi selecionado para um estudo sobre o comportamento da fluorose dentária na Cidade de Tete. 0 estudo será realizado no âmbito da minha dissertação para finalizar o curso de medicina Dentária pela Universidade Zambeze, sob orientação da $\mathrm{Dr}^{2}$. Kirénia Rabilero Salgado.

O estudo consiste mum questionário e observação dos dentes dos participantes, utilizando materinis descartíveis.

Os dados obtidos serão absolutamente confidenciais.

A realização deste estudo é muíto importante para a conclusão do meu curso, pelo que agradeço a sua colaboração.

Por favor, preencha e devolva o destacável

Eu,

encarregado de echucação do/a autorizo a participação do/a meu etucando no estudo para a dissertação acima escrita.

O encarregado de educação:

Eu, aceito participar no estudo para a dissertação acima escrita.

O participante: 
Prevalência e severidade de fluorose dentária na cidade de Tete, Moçambique, no período de 2013

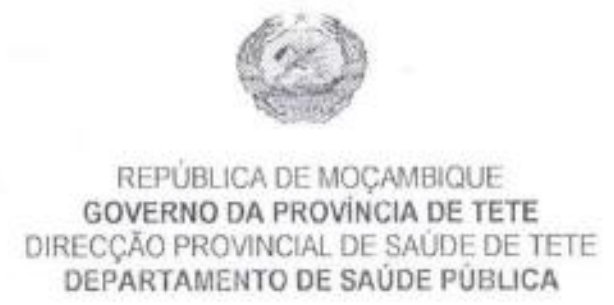

\section{RESULTADOS DO CONTROLO DO NIVEL DO FLUOR NAS FONTES DA CIDADE}

Controlo da Somana 10 a 15 de daneirn de 2011

\begin{tabular}{|c|c|c|c|c|c|}
\hline $\begin{array}{l}N \text { da } \\
\text { ordem }\end{array}$ & Tipo da Fonte & Bsirro & Unidade & $\begin{array}{l}\text { Nivel } \\
\text { do F } \\
\text { (ppm) }\end{array}$ & $\begin{array}{l}\text { Parametros } \\
\text { admissiveis } \\
\text { (ppm) }\end{array}$ \\
\hline 01 & Fontenária Chingale & Francisco Manyange & Chingale & $<1.5$ & 1.5 \\
\hline 02 & Tomoira $\operatorname{CSN}^{\circ} 3$ & Francisco Maryanga & - & $<15$ & 1.5 \\
\hline 03 & Tomaira Casa Agraria & Francisco Marnyanga & Chingaio & 1.2 & 1.5 \\
\hline 04 & ET,A & Josina Machial & - & $<1,5$ & 1.5 \\
\hline 05 & Fontenarita Frescom & Filipe S. Magaia & Nhamatsira & 1.5 & 1.5 \\
\hline 06 & Fontenaria Quinta dus Pornbas & Filipe S. Magaa & Nhamabira & 1.5 & 1.5 \\
\hline 07 & Eontenária Oficina & Sancra Machiel & Ganongola & 1.4 & 1.5 \\
\hline 08 & Deposito asteo do FIPAG & Samora Machiel & - & 1.4 & 1.5 \\
\hline 09 & Fontenária da Sodo do Bairfo & Mpadué & - & 21,5 & 1,5 \\
\hline 10 & Terpreira as Posto des Sinute & Whatin & - & 315 & 3,0 \\
\hline 11 & Fontenaria da Sede do Bairro & Malous S Muthomba & - & 1.4 & 1.5 \\
\hline 12 & Furo Quinta Prisa & Chingodzi & - & 1.5 & 1.5 \\
\hline 13 & Furo a margem do rio Rovobwo & Chirkgodzi & $=$ & 1.4 & 1,5 \\
\hline 14 & Fonlenaria moredado cambinde & Matundo & - & 1.4 & 15 \\
\hline 15 & Rio Zambeze & Matundo & - & 1.3 & 1.5 \\
\hline
\end{tabular}

O Técnico

Calisto Leonarco Duzenta

Tete, 15 de Janeiro de 2011 